\title{
Zur Kenntniss der Xanthorrhoeaharze
}

von

\section{Dr. Max Bamberger.}

Aus dem Laboratorium für allgemeine und analytische Chemie an der $\mathrm{k}, \mathrm{k}$. technischen Hochschule in Wien.

(Vorgelegt in der Sitzung am 18. Mai 1893.)

Die Xanthorrhoeaharze, ${ }^{1}$ die unter den Namen Akaroidharz, Botanybaygummi, Grass - tree Gum, Nuttharz in den Handel gebracht werden, waren schon oft Gegenstand der chemischen Untersuchung.

Bereits im Jahre 1799 untersuchte Lichtenstein ${ }^{2}$ das gelbe Xanthorrhoeaharz, Laugier ${ }^{3}$ fand in demselben Benzoësäure und Bassorin, Trommsdorf ${ }^{4}$ gelang es, dasselbe in zwei verschiedene Harze, Widmann ${ }^{5}$ in drei zu zerlegen.

Stenhouse ${ }^{6}$ wies in dem gelben Harz Zimmtsäure und Benzoësäure nach und erhielt bei der Behandlung desselben mit Salpetersäure grössere Quantitäten Pikrinsäure. Hlasi w etz und $\mathrm{Barth}{ }^{7}$ fanden, dass dieses Harz mit Kali verschmolzen bedeutende Mengen Paraoxybenzoësäure, Resorcin, eine Doppelverbindung von Protocatechusäure mit Paraoxybenzoësäure und Brenzcatechin gibt. Ich habe nun die Xanthorrhoeaharze einer neuerlichen Untersuchung unterzogen und erlaube mir über die Resultate derselben zu berichten.

1 W i esner, Die technisch verwendeten Gummiarten, Harze und Balsame, 189 und Die Rohstoffe des Pflanzenreiches, 148.

2 v. Crell's chemische Annalen, 1800, II, S. Z. 8, 242.

3 Annales de Chimie, 76, 265.

4 Trommsdorf's Taschenbuch, 7, 1.

5 Repertorium für die Pharmacie, 22, 198.

6 Annalen der Chemie und Pharmacie, 57, 84.

7 Ebenda, 139, 78. 


\section{Das gelbe Xanthorrhoeaharz (Xanthorrhoea hastilis Sm.)}

Das zur Untersuchung nöthige Rohmaterial bezog ich von Gehe und Schuchardt, ausserdem hatte Herr Fabrikant Keil die Güte, mir eine grössere Quantität von gelbem und rothem Harz zur Verfügung zu stellen, wofür ich demselben an dieser Stelle bestens danke.

Die Säure-, Verseifungs- und Jodzah1 des Harzes wurde nach der Methode von v. Schmidt und Erban ${ }^{1}$ bestimmt. Die beiden ersten Constanten machen aber auf keine besondere Genauigkeit Anspruch, da die alkoholische Lösung des Harzes beim Behandeln mit Kalilauge stark braun gefärbt wird und dadurch die Titrirung sehr erschwert ist.

Die nachstehenden Zahlen beziehen sich, falls nicht ausdrücklich die Bezeichnung "tohes Harz « angeführt ist, auf das mit Alkohol gereinigte Harz.

$\underbrace{\text { Säurezah1 }}_{\begin{array}{l}133 \\ 132\end{array}} \underbrace{\text { Verseifungszahl }}_{220-225^{2}} \quad \underbrace{\text { Jodzahl }}_{86}$

Die Methylzahl des gelben Akaroidharzes wurde schon seinerzeit von mir ${ }^{3}$ bestimmt und dafür der Werth 26.4 gefunden. Die Bestimmungen derselben wurden nun mit dem Apparat von Benedikt und Grüssner wiederholt und dafür folgende Zahien ermittelt:

$$
\text { Methylzahlen. }
$$

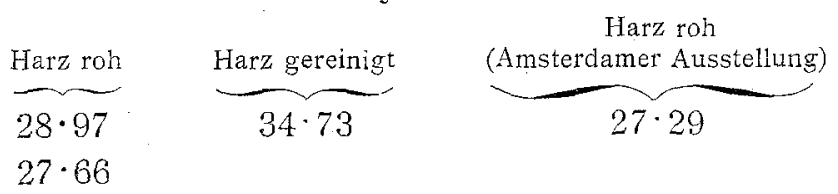

Die Carbonylzahl des gelben Harzes, welche F. Kitt nach der Methode von Strach $e^{4}$ auszuführen die Güte hatte, beträgt in Procenten Carbonylsauerstoff ausgedrückt 0.38 .

1 Monatshefte für Chemie, 655.

2 Die Verseifungszahl verdanke ich einer freundlichen Mittheilung des Herrn Apothekers A. Krem $e$ l.

3 Monatshefte für Chemie, 11, 84.

4 Ebenda, 12, 524. 
Ein Vorversuch ergab, dass die schon bei dem Überwallungsharze ${ }^{1}$ beschriebene Methode des Auskochens desselben mit Wasser auch hier bei den Xanthorrhoeaharzen mit Erfolg anwendbar ist, nachdem eine kleine Quantität Harz, mit Wasser gekocht, an dasselbe eine beträchtliche Menge krystallisirbare Substanz abgab.

Um nun eine grössere Quantität der letzteren herzustellen, wurden circa $1700 \mathrm{~g}$ gelbes Harz mit 5 l 95\% igem Alkohol übergossen und das Unlösliche, das zum grössten Theile aus Rinde bestand und circa $100 \mathrm{~g}$ wog, von der Lösung abfiltrirt. Aus letzterer wurde der Alkohol durch Einleiten von Wasserdampf entfernt und die heisse wässerige Lösung vom geschmolzenen Harze abgegossen. Aus derselben schied sich bei längerem Stehen eine bedeutende Menge krystallisirter Substanz ab, die abfiltrirt wurde. Das Filtrat lieferte, mit Äther ausgeschüttelt, noch bedeutende Mengen von krystallisirter Substanz.

Das zurückbleibende Harz wurde noch 14 mal mit je $4 . l$ Wasser durch 12 Stunden ausgekocht, und bei jeder Auskochung nicht unbeträchtliche Mengen von dieser krystallisirten Substanz gewonnen. Durch Auskochen allein war also an eine vollständige Erschöpfung des Harzes an derselben nicht zu denken, es wurde daher das wiederholt mit Wasser behandelte Harz in $3 \%$ iger Kalilauge gelöst, die heisse Flüssigkeit mit verdünnter Schwefelsäure zersetzt; die Lösung vom ausgeschiedenen Harz abfiltrirt und mehreremale mit Äther ausgeschüttelt. Der Äther hinterliess beim Abdestilliren noch einen reichlichen Rückstand.

Die gesammte bis hieher erhaltene trockene Rohkrystallisation wurde viermal mit Chloroform ausgekocht, um die in derselben enthaltene Zimmtsäure und Benzoësäure sowie das etwa vorhandene Vanillin zu entfernen. Der grösste Theil der Substanz blieb ungelöst. Die von dem Ungelösten $A$ getrennte Chloroformlösung wurde eingedampft der Rückstand $B$ in Äther gelöst und in der später zu beschreibenden Weise untersucht.

1 Monatshefte für Chemie, 12, 441 . 


\section{Aufarbeitung von $A$.}

Der in Chloroform unlösliche Theil wurde in verdünntem heissen Alkohol gelöst, welche Lösung beim Erkalten zu einem krystallinischen Brei erstarte, welcher von der Mutterlauge durch Absaugen getrennt wurde. Die Krystalle wurden dann mehreremale unter Zugabe von Blutkohle mit heissem Wasser umkrystallisirt, hierauf zur vollständigen Reinigung nach der Methode von Tiemann und Herzfeld ${ }^{1}$ in Sodalösung gelöst, dieselbe mit Äther ausgeschüttelt und die zurückbleibende alkalische Lösung dann mit Salzsäure zerlegt. Der ausgeschiedene Niederschlag wurde abfiltrirt und dann mehreremale aus heissem Wasser umkrystallisirt. Aus der erkalteten Lösung schied sich ein in langen Nadeln krystallisirender Körper aus, der bei $206^{\circ}$ schmolz.

Die auf die vorher beschriebene Art gewonnene Verbindung ist eine Säure, welche kohlensaure Salze mit Leichtigkeit zerlegt, leicht löslich in heissem Wasser, Alkohol und Äther, fast unlöslich in Chloroform und Petroleumäther ist. Eisenchlorid gibt mit der alkoholischen Lösung eine dunkelbraune Färbung, Silbernitratlösung wird durch die Säure nicht reducirt.

Die bei $120^{\circ}$ getrocknete Substanz, der Elementaranalyse unterzogen, lieferte nachstehende Resultate:

I. $0 \cdot 3212 g$ Substanz gaben $0.7735 g$ Kohlensäure und $0 \cdot 1392 \mathrm{~g}$ Wasser.

II. $0 \cdot 288 \mathrm{~g}$ Substanz gaben $0 \cdot 695 \mathrm{~g}$ Kohlensäure und $0 \cdot 123 \mathrm{~g}$ Wasser.

In 100 Theilen:

$$
\begin{aligned}
& \text { I. II. } \quad \mathrm{C}_{9} \mathrm{H}_{8} \mathrm{O}_{3} \\
& \text { C....65.67 } 65 \cdot 81 \quad 65 \cdot 85 \\
& \begin{array}{llll}
\mathrm{H} \ldots \ldots . & 4 \cdot 81 & 4 \cdot 74 & 4 \cdot 87
\end{array}
\end{aligned}
$$

Die Wasserbestimmung der aus kaltem Wasser erhaltenen Säure ergab nachstehende Zahlen:

1 Berliner Berichte, 10, 67. 
I. 0.3542 g Substanz gaben $0 \cdot 033$ g Wasser.

II. $0 \cdot 3358 \mathrm{~g}$ Substanz gaben $0 \cdot 0314 \mathrm{~g}$ Wasser.

In 100 Theilen:

$$
\text { Wasser ......9.31 } \quad \text { II. } \quad \frac{\mathrm{C}_{9} \mathrm{H}_{8} \mathrm{O}_{3}+\mathrm{H}_{2} \mathrm{O}}{9 \cdot 89}
$$

Die Resultate der Verbrennungen sowie die Eigenschaften der Säure lassen dieselbe als die auch in dem Überwallungsharz' der Fichte aufgefundene Paracumarsäure erkennen, und zeigte sich dieselbe im Vergleiche zu der von Hlasiwetz dargestellten, in der Sammlung des Laboratoriums vorhandenen, als vollständig identisch.

Obwohl die Paracumarsäure aus sehr verdünnter Lösung auskrystallisirte, dürfte derselben doch etwas wasserfreie Säure beigemengt sein, da die Zahlen für das Wasser zu gering sind.

Die Ausbeute an reiner Paracumarsäure beträgt circa $10 \%$ des angewandten Harzes. Dieselbe stimmt so ziemlich mit der Quantität von Paraoxybenzoësäure überein, welche Hlasiwetz und $\mathrm{B}$ a rth ${ }^{2}$ bei der Kalischmelze aus dem gelben Xanthorrhoeaharz erhielten.

Nachdem nun das gelbe Akaroidharz einen sehr billigen Preis hat ( $1 \mathrm{~K}$. kostet $40 \mathrm{kr}$.), so kann es wohl mit Vortheil zur Darstellung der Paracumarsäure verwendet werden, nachdem nach der bisherigen Bereitungsweise aus Aloë die Ausbeute nach Eigel $l^{3}$ nur $1.5 \%$ beträgt.

Zur weiteren Identificirung wurde noch das Hydroproduct der Paracumarsäure hergestellt, zu welchem Behufe $7 g$ der in Wasser suspendirten Säure mit Natriumamalgam gekocht wurden. Die bei dieser Operation erhaltene Säure zeigte alle Eigenschaften der von $\mathrm{H}$ lasiwe $\mathrm{z}^{4}$ zuerst dargestellten Hydroparacumarsäure. Das Hydroproduct löst sich leicht in Wasser und schmilzt bei $128^{\circ}$.

Die Analyse der bei $100^{\circ}$ getrockneten Substanz gab nachstehendes Resultat:

1 Monatshefte für Chemie, 12, 457 .

2 Annalen der Chemie und Pharmacie, 63, 80.

3 Berliner Berichte, 20, 2528.

4 Annalen der Chemie und Pharmacie, 142, 358. 
$0 \cdot 5034 \mathrm{~g}$ Substanz gaben $1 \cdot 1924 \mathrm{~g}$ Kohlensäure und $0 \cdot 2656 \mathrm{~g}$ Wasser.

In 100 Theilen:

$$
\begin{array}{lr} 
& \begin{array}{r}
\mathrm{C}_{9} \mathrm{H}_{10} \mathrm{O}_{3} \\
\mathrm{C} \ldots \ldots .64 \cdot 60
\end{array} \\
\mathrm{H} \ldots 5 \cdot 06 \\
\hline .5 \cdot 86 & 6 \cdot 02
\end{array}
$$

Sämmtliche eingangs erwähnten Forscher dürften wohl sicher die Paracumarsäure in den Händen gehabt haben, allein sie wurde von denselben nicht als eine neue Säure erkannt, sondern immer mit der gleichzeitig vorhandenen Benzoësäure und Zimmtsäure verwechselt.

\section{Aufarbeitung von $B$.}

Die beim Abdestilliren des Chloroforms zurückbleibende Masse wurde in Äther gelöst und die so gewonnene Lösung behufs Entfernung von etwa vorhandenen Aldehyden mit Natriumbisulfit geschüttelt.

Die weitere Aufarbeitung geschah nach der Methode, welche Tiemann und Haarmann ${ }^{i}$ zur quantitativen Bestimmung des Vanillins in der Vanille angeben.

Die so erhaltenen Aldehyde hatten einen sehr angenehmen, aber nicht an Vanillin erinnernden Geruch und gaben mit Phloroglucin eine intensiv rothe Färbung. Dieselben wurden nun wiederholt mit Petroleumäther (Sp. $105^{\circ}$ ) ausgekocht, bei welcher Behandlung ein Körper in Lösung ging, der sich beim Verdunsten des Lösungsmittels in langen Nadeln ausschied, denen aber noch Krystalle einer anderen Substanz beigemengt waren. Diese Ausscheidung schmolz bei $60^{\circ}$. Aus derselben konnte wohl durch wiederholtes Umkrystallisiren eine Fraction gewonnen werden, die bei $78^{\circ}$ schmolz und eine Methylzahl von 88 hatte, sowie mit Eisenchlorid eine blaue Färbung gab, während das Vanillin bei $81^{\circ}$ schmilzt und eine Methylzahl von 96 gibt. Die Menge der mir zur Verfügung stehenden Substanz war aber so gering, dass ich die Anwesenheit von Vanillin in dem gelben Xanthorrhoeaharz als noch nicht ganz sicher con-

1 Berliner Berichte, 8, 1115. 
statirt haben will. Ich glaube übrigens, dass neben dem etwa vorhandenen Vanillin noch ein anderer methoxylhaltiger Aldehyd anwesend ist, vielleicht $p$-Methoxylsalicylaldehyd.

Ich werde eine grössere Quantität des gelben Harzes auf diese beiden Aldehyde aufarbeiten und hoffe dann Sicheres über die Anwesenheit derselben berichten zu können.

Der bei der Behandlung mit Petroleumäther zurückbleibende Theil löste sich sehr leicht in heissem Wasser. Behufs Reinigung der etwas braun gefärbten Lösung wurde dieselbe mit Blutkohle entfärbt, worauf sich dann beim Abkühlen der Lösung weisse Krystalle ausschieden, die bei $116^{\circ}$ schmolzen, mit Eisenchlorid eine violette Färbung gaben, überhaupt alle Eigenschaften des Paraoxybenzaldehydes zeigten.

Die Analyse der bei $100^{\circ}$ getrockneten Substanz ergab nachstehendes Resultat:

$0 \cdot 165 \mathrm{~g}$ Substanz gaben $0.4166 \mathrm{~g}$ Kohlensäure und $0.072 \mathrm{~g}$ Wasser.

In 100 Theilen:

$$
\begin{array}{r}
\mathrm{C} \ldots \ldots .68 \cdot 85 \\
\mathrm{C} \ldots .+85
\end{array}
$$

Durch Kochen von 1 Theil Paraoxybenzaldehyd mit 3 Theilen Essigsäureanhydrid wurde nach der Vorschrift von Tiemann und Herzfeld ${ }^{1}$ der Essigsäure-Acetylparaoxybenzaldehyd $\mathrm{C}_{6} \mathrm{H}_{4} \backslash \mathrm{O} \backslash \mathrm{C}_{2} \mathrm{C}_{3} \mathrm{O}\left(\mathrm{C}_{2} \mathrm{H}_{3} \mathrm{O}_{2}\right)_{2}$ dargestellt, der in flachen Prismen erhalten wurde, die bei $93^{\circ}$ schmolzen. Leider gestattete die geringe Menge der zur Verfügung stehenden Substanz nicht eine Elementaranalyse auszuführen.

Die durch Ausschütteln mit Natriumbisulfit von den Alde. hyden befreite ätherische Lösung wurde durch Abdestilliren vom Äther befreit. Die zurückbleibende Masse erstarrte zu einem krystallinischen Brei und wurde dann direct, da die Krystalle durch Absaugen nicht gut von der Mutterlauge getrennt

1 Berliner Berichte, 10, 65. 
werden konnten, mit Petroleumäther (Sp. 105\%) mehreremale ausgekocht. Es schied sich aus dem Petroleumäther jedesmal eine reichliche Menge einer etwas gelblich gefärbten krystallinischen Substanz aus.

Um die in dieser Ausscheidung etwa vorhandene Benzoësäure zu gewinnen, wurden die Krystalle längere Zeit in einer Glasschale, die mit einem Uhrglas bedecktwar, auf dem Wasserbade erhitzt. Es sublimirte eine geringe Menge einer Substanz, die bei $120^{\circ}$ schmolz und den charakteristischen Geruch der Benzoësäure zeigte.

Die von letzterer Säure befreite Ausscheidung wurde mehreremale aus Petroleumäther umikrystallisirt, aus welchem sich beim Erkalten schöne weisse Nadeln ausschieden, die bei $133^{\circ}$ schmolzen, leicht in Alkohol, Äther und heissem Wasse löslich waren und mit einer Lösung von Kaliumpermanganat übergossen, einen intensiven Geruch nach Bittermandelöl gaben. Die Säure zeigte also alle Eigenschaften der Zimmtsäure und lieferte, der Elementaranalyse unterzogen, nachstehende Zahlen:

$0 \cdot 4694 \mathrm{~g}$ Substanz gaben $1 \cdot 2522 \mathrm{~g}$ Kohlensäure und $0 \cdot 2522 \mathrm{~g}$ Wasser.

In 100 Theilen:

$$
\begin{array}{lr}
\mathrm{C} \ldots \ldots .72 \cdot 75 & \frac{\mathrm{C}_{9} \mathrm{H}_{8} \mathrm{O}_{2}}{72 \cdot 97} \\
\mathrm{H} \ldots \ldots .5 \cdot 33 & 5 \cdot 40
\end{array}
$$

Die Ausbeute an Zimmtsäure betrug circa 1\% der angewandten Harzmenge.

Zur weiteren Identificirung der Zimmtsäure wurde noch deren Hydroproduct nach der Methode von Gabriel und Zimmermann ${ }^{1}$ dargestellt. Die gewonnene Substanz schmolz bei $49^{\circ}$, zeigte alle Eigenschaften der Hydrozimmtsäure und gab bei der Elementaranalyse folgende Resultate:

$0.467 \mathrm{~g}$ Substanz gaben $1.231 \mathrm{~g}$ Kohlensäure und $0.2764 \mathrm{~g}$ Wasser.

1. Berliner Berichte, 13, 1680. 
In 100 Theilen:

$$
\begin{array}{lr}
\mathrm{C} \ldots \ldots .71 \cdot 89 & \begin{array}{r}
\mathrm{C}_{9} \mathrm{H}_{10} \mathrm{O}_{2} \\
\mathrm{H} \ldots \ldots
\end{array} \quad \begin{array}{r}
72 \cdot 00 \\
6 \cdot 67
\end{array}
\end{array}
$$

Um die neutralen Substanzen im gelben Xanthorrhoeaharz zu bestimmen, wurde $1 K$. in Äther gelöst und die ätherische Lösung wiederholt mit verdünnter Kalilauge ausgeschüttelt. Beim Abdestilliren des Äthers hinterblieb im Kolben ein angenehm riechendes Öl, das eine intensive Phloroglucinreaction gab und nach einiger Zeit einen festen weissen Körper absetzte, der durch Behandeln mit kaltem Alkohol, in dem er fast unlöslich ist, von dem Öle getrennt wurde.

Die so erhaltene weisse Masse, die ein dem Paraffin ähnliches Aussehen zeigte, schmolz bei $75^{\circ}$ und gab bei der Verbrennung $\mathrm{C}=79 \cdot 70 \%$ und $\mathrm{H}=13 \cdot 00 \%$.

Behufs genaueren Studiums der neutralen Substanzen muss ich noch eine grössere Menge des Harzes auf dieselben aufarbeiten.

\section{Das rothe Xanthorrhoeaharz (Xanthorrhoea australis).}

Die Säure- und Verseifungszahl lassen sich beim rothen Harz absolut nicht bestimmen, nachdem die alkoholische Lösung des Harzes intensiv roth gefärbt ist. Die Jodzahl beträgt

\begin{tabular}{|c|c|c|}
\hline \multicolumn{2}{|c|}{ Methylzahlen. } & Carbonylzah \\
\hline Harz (roh) & Harz (gereinigt) & Harz (gereinigt) \\
\hline $\begin{array}{l}60 \cdot 3 \\
60 \cdot 9\end{array}$ & $71 \cdot 12$ & $0 \cdot 97$ \\
\hline
\end{tabular}
in mit Alkohol gereinigtem Harz 105.5.

Die Bestimmung der Methylzahl und Carbonylzahl ergab nachstehende Resultate:

Das rothe Harz wurde ganz analog dem gelben aufgearbeitet. $920 \mathrm{~g}$ Harz wurden in Alkohol gelöst, $80 \mathrm{~g}$ blieben ungelöst. Die erhaltene Säure hatte alle Eigenschaften der Paracumarsäure und schmolz bei $206^{\circ}$. Die Ausbeute betrug circa $2 \%$, also bedeutend weniger als beim gelben Harz. 
Die Analyse der bei $120^{\circ}$ getrockneten Substanz ergab:

$0.3848 g$ Substanz gaben $0.9275 g$ Kohlensäure und $0.166 g$ Wasser.

In 100 Theilen:

$$
\begin{array}{lr} 
& \frac{\mathrm{C}_{9} \mathrm{H}_{8} \mathrm{O}_{3}}{6 . \ldots .65 \cdot 73} \\
\mathrm{H} \ldots . .4 .80 & 65 \cdot 85 \\
4.87
\end{array}
$$

Die Wasserbestimmung ergab:

$0.4242 g$ Substanz gaben $0.0394 g$ Wasser:

In 100 Theilen:

$$
\mathrm{H}_{2} \mathrm{O} \ldots .9 \cdot 30 \% \quad \frac{\mathrm{C}_{9} \mathrm{H}_{8} \mathrm{O}_{3}+\mathrm{H}_{2} \mathrm{O}}{9 \cdot 89}
$$

Zimmtsäure und Benzoësäure konnte ich bis jetzt in dem rothen Harz nicht nachweisen, wohl aber dieselben Aldehyde, die sich im gelben Harz vorfinden. Die Menge des dem Vanillin ähnlichen Aldehyds war aber auch hier so gering; dass an eine genauere Untersuchung desselben nicht gedacht werden konnte.

Der in Petroleumäther unlösliche Theil der Aldehyde wurde leicht durch Wasser aufgenommen, aus welchem sich Krystalle ausschieden, die bei $116^{\circ}$ schmolzen und alle Eigenschaften des Paraoxybenzaldehyds zeigten.

Die Analyse der bei $100^{\circ}$ getrockneten Verbindung ergab: $0.2756 \mathrm{~g}$ Substanz gaben $0.698 \mathrm{~g}$ Kohlensäure und $0.1206 \mathrm{~g}$ Wasser.

In 100 Theilen:

$$
\begin{array}{lr} 
& \\
\mathrm{C} \ldots \ldots 69.04 & \frac{\mathrm{C}_{7} \mathrm{H}_{6} \mathrm{O}_{2}}{68 \cdot 85} \\
\mathrm{H} \ldots \ldots .86 & 4.91
\end{array}
$$

Vorliegende Untersuchung hat somit nachstehende Resultate ergeben: 
1. Durch Auskochen des gelben Xanthorrhoeaharzes mit Wasser wurden circa 10\% Paracumarsäure, 1\% Zimmtsäure, Benzoësäure, ein dem Vanillin ähnlicher Körper und Paraoxy benzaldehyd gewonnen.

2. Das rothe Harz, derselben Behandlung unterworfen, lieferte circa $2 \%$ Paracumarsäure, eine dem Vanillin ähnliche Substanz und Paraoxybenzaldehyd.

Die Untersuchung über die Xanthorrhoeaharze wird fortgesetzt. 